\title{
Turismo sustentable y los diferentes enfoques, aproximaciones y herramientas para su medición.
} Angelica Maria Saeteros Hernandez* Edson Vicente Da Silva** Universidade Federal do Ceará (Brasil)

\author{
Miguel Alfonso Flores Sanchez*** \\ Escuela Politecnica Nacional (Ecuador)
}

\begin{abstract}
Resumen: La sustentabilidad de las actividades económicas ostenta un interés creciente dado que se vincula estrechamente con el ambiente tal es el caso del turismo reconocido como una de las industrias de más rápido crecimiento. La planificación turística se ha convertido en una técnica necesaria para minimizar los impactos que esta pudiera causar. A partir de una conceptualización del turismo sostenible este estudio busca analizar desde la teoría los diferentes enfoques y las herramientas metodológicas para la medición del turismo sostenible y analizar sobre las técnicas más usadas con sus ventajas y desventajas; la investigación concluye que la sustentabilidad turística constituye un concepto no definido por lo que se han hecho diversas aproximaciones para su comprensión y constituye una importante herramienta en la gestión de la sustentabilidad. Para su medición existe el método contable y el analítico; destacando en sus ventajas el método analítico el cual permite obtener una medida global de sustentabilidad del destino, se adapta a territorios locales y permite tomar acciones a los planificadores.
\end{abstract}

Palabras clave: Turismo sustentable; Medición; Métodos; Sustentabilidad.

\section{Sustainable tourism and the differents approaches and tools for its measurement}

Abstract: The sustainability of the economic activities shows an increasing interest given that it is closely related to the environment such is the case of tourism recognized as one of the fastest growing industries. Tourism planning has become a necessary technique to minimize the impacts that this could cause. From a conceptualization of sustainable tourism, this study seeks to analyze from the theory the different approaches and methodological tools for the sustainable tourism measure and analyze the advantages and disadvantages of the most used techniques; The research concludes that sustainable tourism is a not defined concept and different approaches has been done to understand and is an important tool for sustainable management. For its measurement there are the accounting and the analytical method; highlighting in its advantages the analytical method which allows obtaining a global sustainability measure of the destination, adapts to local territories and allows actions to be taken by planners.

Keywords: Sustainable tourism; Measurement; Methods; Sustainability.

\section{Introducción:}

El turismo es reconocido como una actividad económica importante, considerada una excelente alternativa para el desarrollo del destino (Costa, Oliveira, \& Gomes, 2010), sin embargo las actividades económicas son las que causan mayor impacto al ambiente en este sentido la sustentabilidad del turismo se convierte en una exigencia en la sociedad contemporánea, fundamentalmente en aquellas que dependen del ambiente (Acevedo-Gutiérrez, Acevedo, \& Boren, 2011; Browlig \& Gibbon, 2009;

\footnotetext{
* Universidade Federal do Ceará (Brasil); E-mail: angelicasaeteros@yahoo.com

* Universidade Federal do Ceará (Brasil); E-mail: cacauceara@gmail.com

*** miguel.flores@epn.edu.ec
} 
Cunha, 2010; Davidson, 2010; Higham \& Shelton, 2011; Kociolek, A.P., Clair, \& S., 2011; Nelson, 2010; Persha, Agrawal, \& Chhatre, 2011; Wittemeyer, Elsen, Bean, Burton, \& Brashares, 2008), por lo que una gestión deficiente puede convertirlo en un factor de degradación, por ello resulta crucial que los países promuevan políticas que soporten el crecimiento del turismo y la sostenibilidad de esta actividad (UNWTO, 2016a). Este aspecto resulta de vital importancia, pues su creciente desarrollo ha motivado el interés por el análisis de la sustentabilidad, así como el desarrollo de instrumentos y políticas para la planificación y gestión se ha mantenido en aumento tanto en el debate empresarial como en el científico (Sanchs, 2015; Zsögön, 2014).

Así, se constata que planificar el turismo de forma sustentable es la manera más eficaz de evitar que se produzcan daños irreversibles, de minimizar los costos sociales, económicos y ambientales que afectan a los pobladores de las localidades y optimizar los beneficios del desarrollo turístico (Macário de Oliveira \& Pasa Gómez, 2013) a tal punto que desde organismos como la Organización Mundial de Turismo (OMT) se insta a los gobiernos a considerar la sostenibilidad como objetivo global.

Para el logro del turismo sustentable se requiere instrumentos que permitan planificar las actividades desarrolladas en una localidad llevando a consideración los limites de los recursos naturales y su capacidad de renovación (Santos \& Gesinaldo, 2018), además de evaluar el grado de sustentabilidad de la actividad y proponer informaciones necesarias para planificar los destinos (Peral, Lozano, Casas, \& Oyola, 2010), Así, se hace necesario conceptualizar el turismo sustentable y analizar los diferentes enfoques, aproximaciones y herramientas para su medición, a fin de que evalúen las políticas planteadas y proporciones herramienta que permita tomar decisiones para conseguir destinos más sostenibles.

\section{Turismo Sustentable}

Para confrontar los enfoques teóricos sobre el turismo sustentable se debe abordar el amplio debate acerca de la sustentabilidad ya que su definición está en constante evolución y su abordaje varía de acuerdo con el contexto de la región donde se estudia (Chauca Pozo, Soto Aguire, \& López Sánchez, 2016; Hernández Paz, González García, \& Tamez González, 2016).

El vínculo del concepto de desarrollo sustentable con el turismo tuvo lugar en la llamada Río+5 Conferencia, en 1999 y en la Conferencia de Johannesburgo, en el 2002, donde se mencionó la necesidad de un turismo más "habitable", que contribuya al bienestar de las comunidades indígenas y a la conservación de la integridad cultural y ecológica (Ahn, Lee, \& Shafer, 2002; Birch et al., 2011; Bockstaller C., 2003; Boulanger, 2008; Heberlein, Fredman, \& Vuorio, 2002; Manning, 2001; Peace \& Turner, 1990; Pereira, Leadley, Proenc, a, Alkemade, \& Scharlemann, 2010; Santamarina, 2008; UNEP, 2011; Yasarata, Altinay, Burns, \& Okumus, 2010). Así, desde la década de los 90's, el "turismo sustentable" es un concepto que predomina en cualquier discurso sobre el turismo (Lemoine Quintero, Delgado Muñoz, Carvajal Zambrano, \& Alcívar Calderón, 2017; Pérez León, 2010; Van Broeck, 2005).

Fueron varios informes, reuniones y conferencias desarrolladas por organismos internacionales y los gobiernos, encaminados a concebir una definición acertada de turismo sustentable. Entre estos aparece el 41 Congreso de la Asociación Internacional de Expertos Científicos en Turismo (1991), la Cumbre de la Tierra (1992), la Conferencia Euromediterránea sobre Turismo y Desarrollo Sostenible en 1993, la Conferencia Mundial del Turismo Sostenible en Lanzarote (1995), la Agenda 21 para la Industria Turística (OMT, 1995); la Asamblea General de Rio+5 (1997) y, 20 años más tarde, la Conferencia de Desarrollo Sustentable (Rio+20).

El año 2017 fue declarado por las Naciones Unidas como el Año Internacional del Turismo Sostenible, con el objetivo de que el turismo, bien designado y administrado, puede contribuir a las tres dimensiones del desarrollo sostenible ambiental, social y económica (Font, 2017). Esta iniciativa tuvo como finalidad fomentar un cambio en las políticas, las prácticas de empresa y el comportamiento de los consumidores para promover un sector turístico más sostenible (UNWTO, 2017).

Finalmente, en la Vigésima Segunda Reunión de la Asamblea General de la OMT en China (Sept., 2017), se aprobó la Declaración de Chengdu sobre "El turismo y los Objetivos de Desarrollo Sostenible" (ODS). El documento subraya el potencial del sector turístico en términos económicos, sociales y ambientales, e incluye 21 artículos que recomiendan a los Gobiernos a «desarrollar un enfoque integrado y holístico en relación con las políticas turísticas a fin de ampliar la incidencia positiva en el planeta y la prosperidad» Además, propone «llevar a cabo evaluaciones de la contribución del turismo a los ODS y de su compromiso con estos objetivos así como garantizar que el turismo esté presente en las comisiones y grupos de trabajo interministeriales sobre los ODS (UNWTO, 2017). 
Adicionalmente, desde la academia, diversos autores contribuyeron significativamente a la conceptualización del término. Butler (1999) presenta un estudio exhaustivo de las definiciones de este término esbozadas en la última década del siglo pasado. El autor argumenta la necesidad de asegurar que el concepto incluya y sea aplicado tanto al entorno humano como al entorno físico (Butler, 1999), establece que es necesario distinguir los conceptos de turismo sustentable y desarrollo del turismo con principios de sustentabilidad, pues el equilibrio entre generación de procesos de emprendimiento, empleo, utilidades, crecimiento de indicadores de calidad de vida, superación de línea de pobreza (entre otros) y armonía con los recursos, protección de la biodiversidad, conservación de fuentes y áreas especiales se logra en la medida en que se construyan discursos éticos y coherentes, consecuentes con las políticas e instrumentos para hacer empresa y el respeto al medio ambiente, la vida y las futuras generaciones (Murcia García, Valderrama Riveros, \& Morales Valderrama, 2017).

En la Declaración de la Zona de Turismo Sustentable del Caribe (ZTSC), Santo Domingo, abril $16-17,1999$ se estableció que el turismo sustentable

"constituye una respuesta adecuada a los retos que representa incrementar las tasas de crecimiento del empleo y captación de divisas, la protección y preservación del medio ambiente y los recursos naturales, la protección del patrimonio y los valores culturales. Apoyamos la participación comunitaria, así como la participación de intereses locales en aspectos del proceso de desarrollo del turismo, tales como la formulación de políticas, planificación, manejo, propiedad y distribución de los beneficios generados por esta actividad..." (Díaz \& Norman, 2006: 15)

Goeldner y Ritchie (2008) también establecieron que la meta principal del turismo sustentable es proveer experiencias de gran calidad para los visitantes que puedan maximizar los beneficios para los implicados, sin comprometer el entorno, la sociedad, la cultura y la integridad de los destinos. De acuerdo con Font (2017) significa crear beneficios positivos en la triple línea de medioambiente, economía y sociedad.

Como resultado del debate acerca de la definición, la OMT (2005: 14) ha propiciado una definición de turismo sustentable que expone que sus directrices son aplicables a todas las formas de turismo en todos los tipos de destinos, incluidos los de masa y menciona que:

"El turismo sustentable es aquel que tiene plenamente en cuenta las repercusiones actuales y futuras, económicas, sociales y medioambientales para satisfacer las necesidades de los visitantes, de la industria, del entorno y de las comunidades anfitrionas".

El desarrollo sustentable de esta actividad exige la participación informada de todos los interesados relevantes, así como un liderazgo político firme para lograr la colaboración amplia y establecer consenso. El logro del turismo sostenible es un proceso continuo y requiere un seguimiento constante de los impactos, para introducir medidas preventivas o correctivas que resulten necesarias.

El turismo sustentable debe reportar también un alto grado de satisfacción a los turistas y representar para ellos una experiencia significativa, que los haga más conscientes de los problemas de la sostenibilidad y fomente en ellos prácticas turísticas sostenibles.

Así, pues, el turismo sustentable debería:

1) Dar un uso óptimo a los recursos ambientales que son un elemento fundamental del desarrollo turístico, manteniendo los procesos ecológicos esenciales y ayudando a conservar los recursos naturales y la diversidad biológica.

2) Respetar la autenticidad sociocultural de las comunidades anfitrionas, conservar su patrimonio cultural arquitectónico vivo y sus valores tradicionales, y contribuir al entendimiento y a la tolerancia intercultural.

3) Asegurar unas actividades económicas viables a largo plazo, que reporten a todos los interesados unos beneficios socioculturales bien distribuidos, entre los que se cuenten oportunidades de empleo estable y de obtención de ingresos y servicios sociales para las comunidades anfitrionas, y que contribuyan a la reducción de la pobreza.

Como se aprecia, esta constituye una de las más completas y exhaustivas definiciones de turismo sustentable, por cuanto concierne a las tres aristas fundamentales mayormente referenciadas en las definiciones de turismo sustentable (Choi \& Sirakaya, 2006), sin embargo se sugiere que el turismo 
sustentable incluye más dimensiones (Choi \& Sirakaya, 2006) como la ecológica, social, institucional, política, cultural y tecnológica, a nivel internacional, nacional, regional y de comunidad local (Bossell, 1999; Mowforth \& Munt, 1998). Estas, según Colby (1989); Reid (1995) y Slocombe (1993) son consideradas independientes y mutualmente contribuyentes y constituyen la propuesta de Choy y Sirakaya (2006) como dimensiones de la sustentabilidad turística. Otros proponen la económica, social, patrimonial y ética (Díaz \& Norman, 2006), entre otras acepciones.

En ese sentido, se puede afirmar que todas las definiciones y dimensiones asociadas a estas abarcan la totalidad de los sectores de cualquier localidad, y sus relaciones en los diferentes contextos (Pérez, Guerrero, González, Pérez, \& Caballero, 2013), siempre y cuando los principios de sustentabilidad sean aplicados al destinos. Para ello, se han desarrollado varias alternativas para medir el grado de sustentabilidad y a continuación, se hace un recorrido por los existentes en la literatura.

\section{Enfoques para medir la sustentabilidad turística}

La OECD (Organization for Economic Cooperation and Development) ha definido dos grandes enfoques para la medición del desarrollo sostenible: el contable y el analítico (OECD, 2000).

\subsection{El enfoque contable}

Se destacan los Sistemas de Cuentas Nacionales (SCN) una herramienta para medir principalmente el desarrollo económico y el bienestar social este sistema es un marco estadístico que proporciona un conjunto completo, coherente y flexible de información macroeconómicas para la formulación de políticas, análisis y propósitos de investigación.

A partir de que surge el término de desarrollo sostenible se puso de manifiesto que para medir el grado de desarrollo no eran apropiados los SCN (Kee \& de Haan, 2003), lo que propició modificaciones de esta herramienta en el seno de las Naciones Unidas (Clarke \& Islam, 2006) las cuales dieron lugar al Sistema de Cuentas Económicas Medioambientales (SCEM). La nueva herramienta está formada por un conjunto de cuentas que recopilan información de tipo medioambiental y económico, para facilitar el análisis de las interrelaciones entre las actividades económicas y el medio en el que se desarrollan (Kee \& de Haan, 2003).

Tiene como principales objetivos: la segregación y elaboración de todos los flujos y stocks del SCN relacionados con el medio ambiente, la valoración monetaria y en términos físicos de cuestiones ambientales, la evaluación de costos ambientales relacionados con el agotamiento de los recursos naturales y la degradación producto de los procesos de contaminación, y por último, la contabilización de la riqueza nacional, incluyendo los recursos no renovables como capital natural (Blancas Peral, 2009).

Tomando como referencia el SCN, partiendo de la búsqueda de instrumentos para evaluar los impactos económicos del sector, se crearon las cuentas satélites para el turismo, organizando la información sobre los efectos de la actividad turística (Frechtling, 1999). Ha sido establecida a nivel internacional de manera progresiva (Bryan, Jones, \& Munday, 2006) desde la Asamblea General de la OMT, en el año 1983 donde se reconoció la necesidad de estudiar los impactos económicos del turismo en el marco del SCN. En 1994, se definió por esta organización la Cuenta Satélite del Turismo CST como un:

"conjunto de definiciones y clasificaciones integradas en tablas, organizadas en una forma lógica y consistente, que permite contemplar en su totalidad la magnitud económica del turismo, tanto en su aspecto de demanda como en el de oferta" (Izard, Simón, i Hereu, Reverté, \& i de Pineda, 2010, p. 273)

Su objetivo principal es la medición de la demanda y la oferta turística en un territorio, a través de un número variable de cuentas en función de la información disponible, conforme a un sistema internacionalmente convenido, que garantiza su fiabilidad y permite la comparación interterritorial de resultados (Blancas Peral, 2009). Dentro de los aspectos que permite evaluar puede mencionarse: la contribución del turismo al PIB, en la inversión del capital, la productividad de los servicios o el empleo turístico (Bryan et al., 2006; Frechtling, 1999).

Para definir las medidas contables agregadas de la sustentabilidad, se han determinado nuevas medidas a partir del ajuste y adaptación de los agregados tradicionales del SNC, buscando aspectos que permitan realizar una medida más acorde al concepto de desarrollo sostenible. 
A partir del análisis de la literatura realizado por Blancas Peral (2009), se agrupan los nuevos agregados en tres grandes grupos: el primero está conformado por las medidas agregadas de riqueza, definidas para considerar la riqueza natural y las limitaciones e impacto sobre el medioambiente de la actividad económica. Ejemplos: el Producto Nacional Bruto Verde (PNBV), Producto Nacional Neto Verde (PNNV) y el Ahorro Genuino (AG).

El segundo grupo distingue las medidas basadas en la evaluación de los niveles de bienestar de la población, a partir de la incorporación de aspectos de carácter social, institucional y medioambiental determinantes en el bienestar de la población del objeto de estudio (Valentin \& Spangenberg, 2000). Ejemplos: El Índice de Bienestar Económico Sostenible (IBES) o el Indicador de Progreso Genuino (IPG).

Dentro del tercer grupo se ubican las medidas basadas en los impactos y limitaciones de la actividad económica sobre el medio ambiente. A partir de medidas físicas, pueden estimarse las exigencias necesarias para que el medio pueda sostener el consumo de recursos y la generación de residuos sin daños irreversibles. Ejemplo: el Espacio Ambiental, la Huella Ecológica, las medidas de capacidad de carga y la Productividad Neta Primaria (PNP).

Dado el grado de desarrollo de las CST, la Organización Mundial del Turismo ha propuesto un sistema estadístico de medición de la sustentabilidad turística a través de las conexiones entre el marco contable establecido para el turismo, las cuentas satélites del turismo y marco contable del Sistema de Cuentas Económicas y Medioambientales.

La iniciativa busca vincular los beneficios generales que surgen del enfoque contable para asegurar la coherencia interna, la habilidad de comprender brechas de datos y ubicar información diferente en el contexto, y el potencial para derivar indicadores basados en la información económica y medioambiental consistentemente definida (UNWTO, 2016b).

\subsection{El enfoque analítico}

El enfoque analítico es aquel que se basa en el empleo de indicadores para medir la sustentabilidad, según las directrices de la OCDE (2000). Estos, a grandes rasgos, se definen como aquellas características que pueden ser medidas, cuyos niveles absolutos y la dirección en que cambian, tienen como finalidad indicar si la región analizada presenta una situación más o menos sostenible (COM, 1996). Dentro de este enfoque se presupone que una región mejora la sustentabilidad de su proceso de desarrollo si la tendencia de los valores absolutos de sus indicadores refleja una mejora respecto a situaciones anteriores (Romero, Pérez, \& Sande, 2003) o lo que es lo mismo, con respecto a un conjunto de datos o valores establecidos por los tomadores de decisión.

La idea de emplear indicadores para gestionar la sustentabilidad goza de extremada popularidad, por lo que muchos gobiernos y agencias han dedicado recursos sustanciales para el desarrollo y contrastación de indicadores (Bell \& Morse, 2008).

En el contexto de la actividad turística, son conocidos como

"herramientas que permiten conocer tendencias, el cumplimiento de objetivos y brindan la posibilidad de intervenir oportunamente permitiendo una toma de decisiones fundamentada. Cuando están bien concebidos, siguen los cambios durante todo el ciclo de vida del proyecto y son indispensables para una eficaz y lograda gestión, convirtiéndose también en mecanismos de enseñanza y estímulo para alcanzar la sustentabilidad. Ayudan también a la toma de decisiones sobre planificación y a conocer las implicaciones del desarrollo propias del sitio para poder promover la sustentabilidad. Permiten además controlar los impactos económicos, sociales y ambientales a todos los niveles" (Díaz \& Norman, 2006: 20).

Su utilidad ha sido reconocida entre los investigadores por la posibilidad que brindan en la asistencia a los planificadores turísticos y tomadores de decisiones para evaluar actuaciones, establecer metas y en la anticipación a los escenarios futuros (R. Croes, 2011; Crounch, 2011; Dwyer \& Kim, 2003). Estos ayudan en la identificación de cambios y tendencias y pueden emplearse para medir resultados y revelar el desempeño relativo de las entidades (Volo, 2015).

Según la OMT (2005), los indicadores son medidas de la existencia de dificultades o de la gravedad de las ya conocidas, indicios de situaciones o problemas por venir, medidas del riesgo y de la necesidad potencial de acción, y medios para identificar y evaluar los resultados de nuestras acciones. Son conjuntos de información formalmente seleccionada que se utiliza con carácter regular en la medición de los cambios pertinentes para el desarrollo de la gestión del turismo. Pueden medir: a) cambios en 
las propias estructuras turísticas y factores internos; b) cambios en los factores externos que afectan al turismo y c) las repercusiones del turismo.

En el contexto del desarrollo sostenible del turismo, los indicadores son series cronológicas de información estratégica para la sustentabilidad de un destino, sus activos, y en última instancia, el futuro del sector turístico.

En cualquier destino, los mejores indicadores son los que responden a los riesgos y preocupaciones fundamentales respecto de la sustentabilidad del turismo, y facilitan información que puede ayudar a circunscribir problemas y evaluar respuestas. Los indicadores responderán normalmente a asuntos relativos a los recursos naturales y al medioambiente de un destino, a inquietudes respecto de la sustentabilidad económica, a problemas sobre los activos culturales y los valores sociales y, más ampliamente a cuestiones de organización y gestión, tanto en el sector turístico como en el conjunto del destino; además, proporcionan información que sirve para la prevención de problemas en cada una de las dimensiones de la sustentabilidad, para formular estrategias y comunicar ideas (Berke \& Manta, 1999; Lundin, 2003; Singh, Murty, Grupta, \& Dikshit, 2009). Permiten además controlar los impactos económicos, sociales y patrimoniales a todos los niveles (Díaz \& Norman, 2006).

En un proceso de desarrollo sustentable del turismo los indicadores no constituyen un fin en sí mismo, sino herramientas que permiten conocer tendencias, el cumplimiento de objetivos y brindan la posibilidad de intervenir oportunamente permitiendo una toma de decisiones fundamentada. Cuando están bien concebidos, siguen los cambios durante todo el ciclo de vida del proyecto y son indispensables para una eficaz y lograda gestión, convirtiéndose también en mecanismos de enseñanza y estímulo para alcanzar la sustentabilidad. Ayudan también a la toma de decisiones sobre planificación y a conocer las implicaciones del desarrollo propias del sitio para poder promover la sustentabilidad. Permiten además controlar los impactos económicos, sociales y ambientales a todos los niveles (Díaz \& Norman, 2006).

Dada su relevante importancia para medir y gestionar la sustentabilidad turística, se han desarrollado varias iniciativas institucionales y por parte de determinados investigadores para establecer indicadores con la finalidad de medir y gestionar la sustentabilidad turística; para su aplicación existen dos métodos.

El método No agregativo es aquel que propone gestionar la sustentabilidad solo a partir del conjunto de indicadores iniciales seleccionados, sin tener que recurrir a su agregación (Álvarez Díaz \& Valdés Peláez, 2016; Antonini, 2009; Choi \& Sirakaya, 2006; Inskeep, 1991; Lozano-Oyola, Blancas, González, \& Caballero, 2012; Macário de Oliveira \& Pasa Gómez, 2013; Mayer, 2008; Miller, 2001; Pintér, Hardi, \& Bartelmus, 2005; M. d. l. C. Pulido Fernández, 2014; Salinas \& La O, 2006; Torres, Sala Ríos, \& Farré Perdiguer, 2015; Yuri Hanai, 2009).

Dentro de este enfoque sobresalen dos escuelas fundamentales (Bell \& Morse, 2008; Lundin, 2003; Reed, Fraster, \& Dougill, 2006). Los que se basan en la metodología de arriba hacia abajo (top-down) o conocida por expertos (expert led) en la cual los expertos e investigadores definen el concepto que se evalúa y los indicadores para su evaluación en base a sus conocimientos en la materia. Y la escuela basada en la comunidad (community-based) o de abajo hacia arriba (bottom-up), en la cual el proceso de selección de los indicadores se lleva a cabo a partir de las referencias de los implicados en el desarrollo turístico del destino (stakeholders).

Los indicadores que se obtienen de la aplicación de la metodología top-down son recolectados rigurosamente, analizados por expertos y su relevancia es contrastada mediante el empleo de herramientas estadísticas. Sin embargo, este proceso no incluye las percepciones y necesidades de los implicados.

Por otra parte, los indicadores obtenidos mediante el proceso bottom-up son construidos mediante la comprensión del contexto local y se derivan del entendimiento sistemático de las percepciones locales del entorno y la sociedad, lo cual ocasiona que puedan no tener la capacidad de gestionar con precisión la sustentabilidad (Reed et al., 2006).

Independientemente de las diferencias entre las metodologías top-down y bottom-up, existe la posibilidad de integrarlas a partir de las ventajas asociadas a cada una para obtener un procedimiento que sirva de guía para cualquier estudio de sustentabilidad (Reed et al., 2006).

Los Indicadores no agregados miden dimensiones de forma aislada. Como ejemplo se pueden citar los Enviromental Pressure Indicators (EPIs), desarrollados por la Oficina Estadística de la Unión Europea, EUROSTAT, para medir y comparar la sostenibilidad medioambiental entre los países miembros. Este conjunto de 60 indicadores distribuidos en 10 ámbitos de actuación (daños a bosques, presión de la pesca, intensidad del turismo, etc.) fue acordado en el V Programa de Acción Medioambiental. Los seis indicadores simples para cada ámbito pueden, a su vez, agregarse en un índice obteniéndose, por lo tanto, un total de diez índices de presión medioambiental. (De la Huerga Molina, 2014) 
El método agregativo, comprende la obtención de un índice o medida compuesta del concepto bajo análisis, a través de una combinación (o agregación matemática) de los indicadores que representan cada una de sus dimensiones (Nardo, Saisana, Saltelli, \& Tarantola, 2005; OECD, 2008; Saisana, Saltelli, \& Tarantola, 2005; Saisana \& Tarantola, 2002; Saltelli, 2006). De este modo, se obtiene un indicador que resulta ideal para medir conceptos, tales como la sustentabilidad, que son multidimensionales y, por lo tanto, no pueden ser capturados por un indicador simple (Nardo, Saisana, Saltelli, \& Tarantola, 2005; Nardo, Saisana, Saltelli, Tarantola, et al., 2005; OECD, 2008; Saisana et al., 2005; Saisana \& Tarantola, 2002; Saltelli, 2006).

Así, un indicador compuesto (IC) se define como "aquella función matemática de los indicadores parciales que reúne un conjunto de condiciones que se juzgan necesarias para concretar una medición expresiva del objetivo buscado" (Pena, 1978: 67), o lo que es lo mismo, una función de un conjunto de variables e indicadores intermedios, cada una de las cuales contribuye a cuantificar algún aspecto del concepto para el cual se quiere cuantificar su magnitud (López, M., Sánchez, Iglesias, \& P., 2003).

Los IC son ampliamente reconocidos por su utilidad para determinar políticas a seguir, para la comunicación pública (OECD, 2008), etc., proporcionando información acerca del desempeño de los países en campos como el medio ambiente, la sociedad, o el desarrollo tecnológico (SINGH et al., 2009).

De este modo, los autores aseveran que la literatura en la temática de IC revela numerosos conceptos comunes. Entre ellos:

\section{Tabla N. 1: Consideraciones en la utilización de Indicadores Compuesto.}

\begin{tabular}{|c|c|}
\hline Diferencias en la definición & $\begin{array}{l}\text { Dificultad en su definición, pues no existe un término universal y } \\
\text { preciso de sustentabilidad turística }\end{array}$ \\
\hline Complejidad del constructo & $\begin{array}{l}\text { La multidimensionalidad afecta seriamente la calidad y utilidad de los } \\
\text { indicadores obtenidos, ya que apenas una medida puede ser más nítida } \\
\text { que el concepto que pretende cuantificar (D. Mendola, Contu, Oliveri, \& } \\
\text { Burgio, 2013). }\end{array}$ \\
\hline $\begin{array}{l}\text { Retos en la selección de los } \\
\text { componentes / atributos } \\
\text { relevantes }\end{array}$ & $\begin{array}{l}\text { El uso de componentes no relevantes puede crear problemas en la } \\
\text { calidad del indicador y de su carácter predictivo. Los indicadores que } \\
\text { contienen variables débilmente relacionadas con el concepto podrían } \\
\text { aportar información engañosa (Daria Mendola \& Volo, 2017) y llevar a } \\
\text { decisiones erróneas en la administración del destino. }\end{array}$ \\
\hline $\begin{array}{l}\text { Variedad de tipos de destino y } \\
\text { de mercados }\end{array}$ & $\begin{array}{l}\text { La variedad de destinos en tamaño, localización, desarrollo económico y } \\
\text { social, etc. implica limitaciones en el proceso de construcción de los IC y } \\
\text { los rankings de sustentabilidad. }\end{array}$ \\
\hline $\begin{array}{l}\text { Adecuación del procedimiento } \\
\text { de construcción del indicador }\end{array}$ & $\begin{array}{l}\text { Muchos de los estudios realizados proporcionan escasa evidencia de } \\
\text { haber seguido avances estadísticos en la creación de los índices. Como } \\
\text { consecuencia, en investigaciones previas (R. Croes, 2011; Lall, 2001; } \\
\text { Mazanec \& Ring, 2011) los procedimientos metodológicos de diversos } \\
\text { IC son inapropiados o inciertos. Entre los mayores problemas aparecen } \\
\text { los } \\
\text { relacionados con la imputación de datos perdidos, efectos de causalidad } \\
\text { erróneos, ponderación arbitraria de las dimensiones y falta de } \\
\text { verificación de su robustez y sensibilidad (Daria Mendola \& Volo, 2017). }\end{array}$ \\
\hline Problemas de datos & $\begin{array}{l}\text { La disponibilidad, accesibilidad a tiempo, fiabilidad, y comparabilidad } \\
\text { de los datos son cruciales en la creación de IC (Robertico Croes \& } \\
\text { Kubickova, 2013; Mazanec \& Ring, 2011). La calidad estadística y el } \\
\text { carácter explicativo del IC dependen de la calidad interna de los datos } \\
\text { estadísticos empleados (Daria Mendola \& Volo, 2017). }\end{array}$ \\
\hline $\begin{array}{l}\text { Utilidad de gestión del IC } \\
\text { resultante }\end{array}$ & $\begin{array}{l}\text { Las decisiones metodológicas que emergen de la literatura en la } \\
\text { construcción de los IC hacen preocuparse acerca del real poder } \\
\text { informativo de estos índices y permiten concluir que los tomadores de } \\
\text { decisión en el turismo realmente se van a beneficiar de su utilización. }\end{array}$ \\
\hline
\end{tabular}

Fuente: Elaboración propia 
En general, una medida cuantitativa de sustentabilidad del turismo resulta abstracta, debido a las dificultades en cuanto a definición, qué incluir, la contabilización, la comparación de diferentes impactos en términos medibles (Buckley, 2012); y el análisis a través de mecanismos sociales y políticos (Honey, 1999).

\section{Análisis de los enfoques para la medición de la sustentabilidad}

De los métodos encontrados en la bibliografía podemos ultimar que el enfoque contable tiene su ventaja en ofrecer un marco de análisis consistente internamente y ampliamente aceptado, definido sobre una base teórica bien establecida (OECD, 2000). A través de su estructura pueden analizarse las relaciones entre diversos aspectos de la sustentabilidad, obteniendo valoraciones monetarias o físicos para establecer una comparación de las dimensiones del concepto y determinar su importancia relativa. Si se vinculan además con la infraestructura estadística se pueden obtener medidas comparables internacionalmente. Mientras, la aceptación internacional de su marco de análisis facilita la obtención de recursos financieros y propicia la reducción de la toma de decisiones subjetivas para obtener una medida de sustentabilidad.

Sin embargo este método ha frenado su aplicación ya que tiene un complejo proceso de elaboración, utiliza muchos recursos para su implementación, y su metodología es de difícil comprensión por su terminología empleada, unido al requerimiento de un gran número de datos y encuestas que permita brindar información creíble y confiable (Sharma \& Olsen, 2005), por lo que la cuantificación de sus componentes requiere condensar una gran cantidad de información estadística y adoptar decisiones subjetivas en función de la región y período analizados, limitando su aplicación para realizar un análisis interterritorial, ya que la mayoría de estas herramientas han sido diseñadas para ser utilizadas a nivel nacional o regional (Mitchell, 1996).

Cabe destacarse también las dificultades teóricas y prácticas cuando se requiere valorar bienes y flujos ambientales y los capitales humano y social, debido a la ausencia de datos estadísticos adecuados para su estimación. Al prevalecer la perspectiva económica, impide analizar todos los aspectos del desarrollo sostenible, por lo cual, las medidas agregadas se encuentran definidas a una determinada dimensión, que provoca la falta de un análisis multidimensional de la sustentabilidad.

Otra desventaja radica en la falta de consenso en relación a la estimación de sus componentes, afectando el análisis de la sustentabilidad para niveles territoriales locales (Mitchel, 1999). Debido a esta limitante, son escasos los trabajos que definen medidas contables de la sustentabilidad turística, puesto que mayormente se centran en aspectos económicos.

Por tanto, los instrumentos anteriormente mencionados no proporcionan medidas apropiadas de sustentabilidad turística debido a la necesidad de instrumentos que proporcionen una evaluación multidimensional adecuada para el destino turístico.

Para ello aparecen en la literatura en enfoque analítico para lo cuál se seleccionan los indicadores que deben cumplir dos criterios (Reed et al., 2006). Primero, que midan de forma precisa y objetiva el progreso hacia el objetivo fijado y que puedan ser aplicados por los usuarios finales. Estos dos criterios establecen una serie de subcriterios acerca de la objetividad y el uso de los indicadores enmarcados en el estudio realizado por Reed et al (2006) entre los que se mencionan la fiabilidad, representatividad, robustez científica, etc., este enfoque permite considerar las valoraciones de los implicados en la definición de los indicadores, vienen determinados por los objetivos de los mismos y las preferencias de los analistas, lo que pone de manifiesto la inexistencia de un único procedimiento metodológico (Peral et al., 2010).

Bajo este enfoque se puede utilizar el método no agregativo al análisis de la sustentabilidad, donde autores consideran que se debe detener el estudio una vez que se tiene el conjunto de indicadores y no dar un paso más allá, que consistiría en crear un indicador compuesto o una medida agregada de sustentabilidad la razón principal radica en el empleo de juicios arbitrarios en el proceso de agregación, los cuales responderán a las características del analista o el decisor, por lo tanto, influyen en el valor de la medida obtenida (Nardo, Saisana, Saltelli, Tarantola, et al., 2005; OECD, 2008; Sharpe, 2004).

Este método resulta de difícil aplicación por el amplio número de indicadores. Adicionalmente resulta complejo el establecimiento de comparaciones entre destinos, o de un mismo destino en diferentes períodos.

Por ello, frente a estas dificultades, aparece la opción de agregar la información contenida en el sistema inicial de indicadores en una medida que sea capaz de reflejar la situación de cada una de las unidades evaluadas con respecto al concepto que se pretende medir. 
Su característica principal constituye su habilidad para resumir, enfocar y condensar la enorme complejidad del entorno que resulta tan dinámico, en una cantidad de información, que resulta fácilmente manejable (Godfrey \& Todd, 2001). En fin, estos simplifican, cuantifican, analizan y comunican información compleja (Warhurst, 2002). Estas características los convierten en herramientas útiles para la toma de decisiones, de ahí su aplicabilidad y, por ende, el amplio uso que tienen en la actualidad (J. I. Pulido Fernández, 2009).

En lo particular, los IC son más fáciles de interpretar que tratar de encontrar un comportamiento común en un conjunto de indicadores separados (Nardo, Saisana, Saltelli, \& Tarantola, 2005). Dependiendo del número de indicadores o de "variables de entrada", estos se clasifican en (a) individuales o simples, en otras palabras, basados en un solo indicador simple "o inicial" o (b) compuestos, que significa que el índice se basa en dos o más indicadores iniciales. De este modo, mediante la combinación de información rica y relevante en un simple valor, los IC proveen a los tomadores de decisiones económicas y políticas de una figura holística del fenómeno bajo observación (Saltelli, 2006, 2007).

La idea de emplear indicadores para gestionar la sustentabilidad goza de extremada popularidad, por lo que muchos gobiernos y agencias han dedicado recursos sustanciales para el desarrollo y contrastación de indicadores (Bell \& Morse, 2008).

$\mathrm{Su}$ importancia se complementa con el hecho de que sobresalen entre las alternativas adoptadas para medir la sustentabilidad, al constituir herramientas de evaluación que permiten chequear cuán exitosas han sido las acciones y políticas seleccionadas (Bank, 1997; Farsari \& Prastacos, 2002; Hardi \& Barg, 1997; U.N., 1998) y si se está actuando adecuadamente en el camino hacia la sustentabilidad del destino.

Se destacan por su fiabilidad para describir situaciones, identificar problemas potenciales y supervisar y evaluar las acciones tomadas (Bank, 1997; Hardi \& Barg, 1997; U.N., 1998); además, proporcionan información que sirve para la prevención de problemas en cada una de las dimensiones de la sustentabilidad, para formular estrategias y comunicar ideas (Berke \& Manta, 1999; Lundin, 2003; Singh et al., 2009).

En la literatura se registran abundantes iniciativas para gestionar la sustentabilidad turística a través de este método. Entre ellas, la OMT, en 1997, con la presentación de la "Guía práctica para el Desarrollo y uso de Indicadores de Turismo Sostenible”, expone cómo utilizar diez indicadores ambientales clave y una serie de indicadores de sitios específicos en zonas costeras, regiones montañosas, comunidades tradicionales e islas pequeñas. Además, incluye un estudio piloto de estos indicadores en Canadá, México, Argentina y los Estados Unidos (OMT, 1997).

Otras organizaciones internacionales han hecho sus contribuciones en ese sentido, como la Oficina Europea de Estadísticas (EUROSTAT), la OCDE y la Comisión Europea, que entre 2004 y 2006 acometieron el "Trabajo Metodológico para medir la Sustentabilidad Turística" y, como resultado, crearon el "Sistema Europeo de Indicadores Turísticos (ESTI) para la Gestión Sustentable de los Destinos", con un conjunto de 20 indicadores clave de turismo sustentable, incluyendo la descripción detallada de cada uno. Estos forman la base de los indicadores que pueden ser empleados a nivel nacional, regional y local en los países europeos y a nivel de comunidades (COM, 2006).

En términos de medición y propuesta de indicadores de turismo sustentable desde la OMT, la mayor contribución se registra en 2005, con el relevante trabajo correspondiente a la "Guía Práctica de Indicadores de Desarrollo Sostenible para los Destinos Turísticos" construida a partir de los trabajos previos desarrollados en la misma temática. Esta incluye alrededor de 700 indicadores a través de 13 temas fundamentales (UNWTO, 2016b).

En ese mismo orden, el Programa de las Naciones Unidas para el Medio Ambiente (PNUMA) y la OMT presentaron en 2006 la "Guía práctica para Responsables Políticos: Por un Turismo más Sostenible" que abarca diferentes aspectos relativos a la sustentabilidad. Se identificaron las políticas e instrumentos específicos aplicados en territorios que han contribuido eficazmente a hacer del turismo un sector más sustentable, a partir de las experiencias en casos reales recogidos por diversas partes del mundo, los cuales han logrado tener éxito en la consecución de los objetivos del desarrollo sostenible (OMT \& PNUMA, 2006).

También se realizaron algunas iniciativas regionales como la Comunidad Andina, que desarrolló el Taller Regional para Países Andinos sobre Indicadores de Sustentabilidad en Destinos Turísticos en 2005, con la determinación de las líneas de acción para implementar los indicadores de sustentabilidad en los destinos del territorio. Así, siguiendo las directrices de la OMT (2005), se identificaron los indicadores para gestionar la sustentabilidad de un destino en cuestión. Se escogió Rurrenabaque, Bolivia, a modo de ejemplo. Adicionalmente, aparece el Programa STEP- Región Andina Y América del Sur "Turismo 
Sostenible y Erradicación de la Pobreza", auspiciado por la OMT, dentro del cual se desarrollaron varios proyectos y se identificaron indicadores para gestionar la sustentabilidad turística en estos países.

Adicionalmente, en el marco de la Asociación de Estados del Caribe, con el "Manual de Procedimientos para Entrenadores en Turismo Sustentable" (Díaz \& Norman, 2006), a partir de materiales elaborados por la Agencia Francesa de Ingeniería Turística (AFIT) y la Consultoría Francesa Geo-System (AFIT-Geosystem), la OMT, la Organización de Turismo del Caribe (CTO), la Alianza Caribeña para el Turismo Sustentable (CAST), y otros preparados por Organizaciones especializadas en Turismo del Caribe. Se proponen 69 indicadores, entre normativos y locales, para medir y gestionar la sustentabilidad del turismo en la región del Caribe.

A pesar de la utilidad de este enfoque, es preciso mencionar que cuenta con algunas limitaciones (Zarzosa, 1996; Zarzosa et al., 2005). Entre ellas aparece la ambigüedad del significado de cada indicador, pues no siempre resulta sencillo interpretar los resultados obtenidos para cada uno en un mismo sentido. Este problema se puede solventar mediante la correcta definición de los aspectos teóricos que se miden y la consideración de las combinaciones de los valores de los indicadores durante la interpretación de los resultados.

Otra debilidad reside en la escasez de los datos estadísticos en diversos destinos a analizar, por lo que la construcción de la base de datos para el análisis debe ser desarrollada cuidadosamente. La otra limitante se refiere a la heterogeneidad de las fuentes de información. Por ello, en la medida de lo posible, se recomienda emplear siempre la misma fuente para cada indicador.

\section{Conclusión}

En conclusión, podemos dirimir que la utilidad del enfoque analítico y la agregación desde la teoría es un método adecuado para medir la sustentabilidad turística ya que está avalada, se ajusta plenamente al concepto para el cuál fueron seleccionados, su aplicación ha tenido buena aceptación y permiten aprovechar sus ventajas, entre las que sobresale que la medida o IC obtenido se ajusta a nivel de análisis territorial y se compone de elementos fácilmente comprensibles por el usuario final. Ello contribuye a garantizar la utilidad de estos instrumentos en la gestión de los destinos turísticos de forma sustentable y la posibilidad de influenciar las decisiones tomadas a interpretar los resultados. Además, la medida puede aplicarse a niveles administrativos que, de forma desagregada, permiten realizar análisis comparativos territoriales o temporales, en caso de tratarse de la gestión de la sustentabilidad turística en un solo territorio.

En resumen, este enfoque resulta apropiado para crear índices de gestión de la sustentabilidad turística en el destino propuesto por cuanto presenta innumerables ventajas y sus puntos débiles pueden ser salvados mediante el empleo de aproximaciones y una rigurosa selección de los indicadores a emplear y de sus calores correspondientes.

\section{Bibliografía}

Acevedo-Gutiérrez, A., Acevedo, L., \& Boren, L. 2011. Effects of the presence of official-looking volunteers on harassment of New Zealand fur seals. Conservation Biology, 25(3), 623-627.

Ahn, B. Y., Lee, B. K., \& Shafer, S. 2002. Operationalizings sustainabilityin regional tourism planning: an aplication of the limits of acceptable change framework. Tourism Management, 23, 1-15.

Álvarez Díaz, R., \& Valdés Peláez, L. 2016. Selección e indicadores para la evaluación del desarrollo sostenible de un destino turístico. Aplicación al municipio de Gijón. ROTUR, Revista de Ocio y Turismo, 11, 12-21.

Antonini, A. 2009. La medida de la sostenibilidad de la ciudad histórico-turística. (Tesis doctoral), Universitat Politécnica de Catalunya.

Bank, W. 1997. Expanding the measure of wealth. Indicators of environmentally sustainable development. Retrieved from http://www-esd.worldbank.org

Bell, S., \& Morse, S. 2008. Sustainability indicators: Measuring the Immeasurable (V. Sterling Ed.). London, UK: Earthscan.

Berke, P., \& Manta, M. 1999. Planning for sustainable development: measuring progress in plans. Retrieved from Cambrige, Massachusetts: http://www.lincolninst.edu/pubs/pubdetail 
Birch, E., Lynch, A., Andreason, S., Eisenman, T., Robinson, J., \& Steif, K. 2011. Measuring U.S. Sustainable Urban Development. Philadelphia, U.S.A.

Blancas Peral, F. J. 2009. Indicadores sintéticos de turismo sostenible: una aplicación para los destinos turísticos de Andalucía. (Memoria para optar al Grado de Doctor por la Universidad Pablo de Olavide de Sevilla), Universidad Pablo de Olavide, Febrero de 2009.

Bockstaller C., G. P. 2003. How to validate environmental indicators. Agricultural Systems, ELSEVIER, $76,639-653$.

Bossell, H. 1999. Indicators for sustainable development: Theory, method and application. Winnipeg, Canada.

Boulanger, P. M. 2008. Sustainable development indicators: a scientific challenge, a democratic issue. S. A. P. I. E. N. S., 1, 15.

Browlig, S., \& Gibbon, P. 2009. Counting Carbon in the Marketplace: Part I-Overview Paper. [Paper presented at the OECD 2009 Global Forum on Trade: Trade and Climate Change]. Paris.

Bryan, J., Jones, C., \& Munday, M. 2006. The Contribution of Tourism to the UK Economy: Satellite Account Perspectives. The Service Industries Journal, 26(5), 493-511.

Buckley, R. 2012. Sustainable tourism: research and reality. Annals of Tourism Research, 39(2), 18. doi:10.1016/j.annals.2012.02.003

Butler, R. W. 1999. Sustainable tourism: a state-of-the-art review. Tourism Geographies, 1(1), 7-25. doi:10.1080/14616689908721291

Chauca Pozo, C. A., Soto Aguire, R. L., \& López Sánchez, R. R. 2016. Modelo de turismo sostenible para la no dependencia petrolera en el Yasuní. Revista Publicando, 3(7), 220-235.

Choi, H. C., \& Sirakaya, E. 2006. Sustainability indicators for managing community tourism. Tourism Management, 27, 1274-1289.

Clarke, M., \& Islam, S. M. N. 2006. National Account Measures and Sustainability Objectives: Present Approaches and Future Prospects Sustainable Development, 14, 219-233.

Colby, M. 1989. The evolution of paradigms of environmental management in development. Retrieved from Washington DC: The World Bank:

COM. 1996. Ciudades Europeas Sostenibles. Informe. Retrieved from Bruselas:

COM. 2006. Methodological work en measuring the sustainable development of tourism. Part 2: Manual on sustainable development indicators of tourism. Retrieved from

Costa, C. C., Oliveira, I. S. S., \& Gomes, L. J. 2010. Percepción ambiental como estrategia para el ecoturismo en unidades de conservación. Estudios y perspectivas en turismo, 19(6), 1105-1120.

Croes, R. 2011. Measuring and explaining competitiveness in the context of small island destinations. Journal of Travel Research, 50(4), 431-442. doi:10.1177/0047287510368139

Croes, R., \& Kubickova, M. 2013. From potential to ability to compete: Towards a performance-based tourism competitiveness index. Journal of Destination Marketing \& Management, 2, 146-154.

Crounch, G. I. 2011. Destination competitiveness: An analysis of determinant attributes. Journal of Travel Research, 50(1), 27-45.

Cunha, A. A. 2010. Negative effects of tourism in a Brazilian Atlantic forest national park. Journal for Nature Conservation, 18(4), 291-295.

Davidson, D. J. 2010. The Applicability of the Concept of Resilience to Social Systems: Some Sources of Optimism and Nagging Doubts. Society and Natural Resources, 23(12), 1135-1349.

De la Huerga Molina, E. 2014. Medición de la sostenibilidad en el sector turístico.

Díaz, G., \& Norman, A. 2006. Manual de Procedimientos para Entrenadores en Turismo Sustentable.

Dwyer, L., \& Kim, C. 2003. Destination Competitiveness: Determinants and indicators. Current Issues in Tourism, 6(5), 369-414.

Farsari, Y., \& Prastacos, P. 2002. Sustainable Development Indicators: An overview Retrieved from http:http://www.iac.mforth.gr/regional/papers/Asteras-English

Font, X. 2017. What meaning for sustainability? Creating tourism impacts in a slippery policy context. Journal of Policy Research in Tourism, Leisure and Events, 9(2), 209-215. doi:10.1080/19407963.2 016.1258514

Frechtling, D. C. 1999. The Tourism Satellite Account: Foundations, Progress and Issues. Tourism Management, 20, 163-170.

Godfrey, L., \& Todd, C. 2001. Defining Thresholds for Freshwater Sustainability Indicators within the Context of South African Water Resource Management. Paper presented at the 2nd WARFA/Waternet Symposium: Integrated Water Resource Management: Theory, Practice, Cases, Cape Town, South Africa. http://www.waternetonline.ihe.nl/ 
Goeldner, C. R., \& Ritchie, J. 2008. Tourism: Principles, Practices, Philosophies. 11th edn. Wiley: New Jersey.

Hardi, P., \& Barg, S. 1997. Measuring Sustainable Development: Review of Current Practice. Occasional Paper(17).

Heberlein, T. A., Fredman, P., \& Vuorio, T. 2002. Current tourism patterns in the Swedish mountain region. Mountain Research and Development, 22(2), 142-149.

Hernández Paz, A., González García, H., \& Tamez González, G. 2016. Desarrollo sustentable: De la teoría a la práctica. Nuevo León, Monterrey, México: Facultad de Ciencias Políticas y Administración Pública, Universidad Autónoma de Nuevo León.

Higham, \& Shelton. 2011. Tourism and wildlife habituation: Reduced population fitness or cessation of impact? Tourism Management, 32(6), 1290-1298.

Honey, M. 1999. Ecotourism and sustainable development. Washington DC: Island.

Inskeep, E. L. 1991. Tourism planning: an integrated and sustainable development approach. . Van Nostrand Reinhold, New York.

Izard, O. M., Simón, R. A., i Hereu, J. C., Reverté, F. G., \& i de Pineda, J. M. P. 2010. Gestión pública del turismo (Vol. 159): Editorial UOC.

Kee, P., \& de Haan, M. 2003. Accounting for Sustainable Development. Paper presented at the Workshop on accounting frameworks to measure sustainable development, Paris.

Kociolek, A.P., C., Clair, S., \& S., P. D. 2011. Effects of road networks on bird populations. Conservation Biology, 25(2), 241-249.

Lall, S. 2001. Competitiveness indices and developing countries: An economic evaluation of the global competitiveness report. World Development, 29(9), 1501-1525.

Lemoine Quintero, F. Á., Delgado Muñoz, K. E., Carvajal Zambrano, G. V., \& Alcívar Calderón, V. E. 2017. La percepción sobre sustentabilidad del turismo en el Cantón San Vicente. Revista Magazine de las Ciencias, 23-39.

López, V., M., E., Sánchez, F., Iglesias, P. e., \& P., J. L. 2003. Bienestar socioeconómico de los municipios gallegos. Revista Galega de Economía, 12(2), 1-24.

Lozano-Oyola, M., Blancas, F. J., González, M., \& Caballero, R. 2012. Sustainable tourism indicators as planning tools in cultural destinations. Ecological Indicators, 18, 659-675.

Lundin, U. 2003. Indicators for measuring the sustainability of urban water systems-a life cycle approach. (PhD Thesis), Chalmers University of Technology, Göteborg, Sweden.

Macário de Oliveira, V., \& Pasa Gómez, C. 2013. Indicadores de sustentabilidad para la actividad turística. Una propuesta de monitoreo usando criterios de análisis. Estudios y Perspectivas en Turismo, 22, 177-197.

Manning, R. 2001. Visitor experience and resource protection: a framework for managing the carrying capactity of National Parks. Journal of Park and Recreation Administration, 19(1), 93-108.

Mayer, A. L. 2008. Strengths and weaknesses of common sustainability indices for multidimensional systems. Environmental International, 34, 277-291. doi:10.1016/j.envint.2007.09.004

Mazanec, J. A., \& Ring, A. 2011. Tourism destination competitiveness: Second thoughts on the world economic forum reports. Tourism Economics, 17(4), 725-751.

Mendola, D., Contu, G., Oliveri, A. M., \& Burgio, M. 2013. La competitivitá di una destinazione turistica. In A. M. Oliveri \& S. De Cantis (Eds.), Mobilit a del turismo regionale incoming. Aspetti socio-economici dei comportamenti e delle motivazioni (pp. 107-118). Millan: McGraw-Hill Education.

Mendola, D., \& Volo, S. 2017. Building composite indicators in tourism studies: Measurements and applications in tourism destination competitiveness. Tourism Management, 59, 541-553. doi:0.1016/j. tourman.2016.08.011.

Miller, G. 2001. The development of indicators for sustainable tourism: results of a Delphi survey of tourism researchers. Tourism Management, 22, 351-362.

Mitchel, G. 1999. Problems and Fundamentals of Sustainable Development Indicators. Sustainable Development, 4, 1-11.

Mowforth, M., \& Munt, I. 1998. Tourism and sustainability. Development and new tourism in the third world. 2nd edn. Routledge, London.

Murcia García, C., Valderrama Riveros, O. C., \& Morales Valderrama, A. 2017. Turismo sostenible: una conceptualización de su viabilidad para el municipio de Ibagué, desde un estado del arte pertinente.

Nardo, Saisana, M., Saltelli, A., \& Tarantola, S. 2005. Tools for composite indicators building. Retrieved from http://farmweb.jrc.cec.eu.int/ci/bibliography.htm 
Nardo, Saisana, M., Saltelli, A., Tarantola, S., Hoffman, A., \& Giovanni, E. 2005. Handbook on constructing composite indicators: Methodology and user guide: OECD Publishing.

Nelson, F. 2010. Community rights, conservation and contested land: The politics of natural resource governance in Africa. Earthscan, London.

OECD. 2000. Frameworks to Measure Sustainable Development. Paris: Organisation for Economic Co-operation and Development.

OECD. 2008. Handbook on constructing composite indicators: Methodology and user guide. Organization for Economic Co-operation and Development: Joint Research Center, European Commission.

OMT. 1995. Lo que todo gestor turístico debe saber. Guía práctica para el desarrollo y uso de indicadores de turismo sostenible. Madrid.

OMT. 1997. Desarrollo turístico sostenible. Guía para los planificadores locales. Madrid.

OMT. 2005. Indicadores de desarrollo sostenible para los destinos turísticos. Guía práctica Retrieved from http://www.e-unwto.org/doi/book/10.18111/9789284408382

OMT, \& PNUMA. 2006. Por un turismo más sostenible. Guía para responsables políticos: Organización Mundial del Turismo.

Organization, W. T. 2004. Indicators of sustainable development for tourism destinations: a guidebook. Peace, D., \& Turner, K. 1990. Economics of natural resources and the environment. Baltimore, U.S.A.

Pena, J. A. (1978). La distancia P: un método para la medición del bienestar. Revista española de Economía, 8, 49-89.

Peral, F. J. B., Lozano, M. G., Casas, F. M. G., \& Oyola, M. L. 2010. Indicadores sintéticos de turismo sostenible: una aplicación para los destinos turísticos de Andalucía. Rect@: Revista Electrónica de Comunicaciones y Trabajos de ASEPUMA(11), 85-118.

Pereira, H. M., Leadley, P. W., Proenc, a, V., Alkemade, R., \& Scharlemann, J. P. W. 2010. Scenarios for global biodiversity in the 21st century. Science, 330 .

Pérez León, V. 2010. Procedimiento de agregación para la construcción de indicadores sintéticos de sostenibilidad en las zonas de turismo de naturaleza en Cuba. (Tesis presentada en opción al título de Doctor en Ciencias Económicas), Universidad de Pinar del Río.

Pérez, V., Guerrero, F., González, M., Pérez, F., \& Caballero, R. 2013. Composite indicator for the assessment of sustainability: The case of Cuban nature-based tourism destinations. Ecological Indicators, 29, 316-324.

Persha, L., Agrawal, A., \& Chhatre, A. 2011. Social and ecological synergy. Science, 331, 1606-1608.

Pintér, L., Hardi, P., \& Bartelmus, P. 2005. Sustainable Development Indicators. Proposals for a Way Forward. Paper presented at the Discussion Paper Prepared for the United Nations Division for Sustainable Development (UN-DSD), International Institute for Sustainable Development

Pulido Fernández, J. I. 2009. Measuring tourism sustainability: proposal for a composite index. Tourism Economics, 15(2), 277-296.

Pulido Fernández, M. d. 1. C. 2014. Metodología para la implantación de la gobernanza como herramienta de gestión de destinos turísticos. (Tesis doctoral), Universidad de Jaén.

Reed, S., Fraster, E., \& Dougill, A. 2006. An adaptive learning process for developing and applying sustainability indicators with local communities. Ecological Economics, 59, 406-418.

Reid, D. 1995. Sustainable development: An introductory guide. Earthscan Publications. London.

Romero, E. M., Pérez, F. M., \& Sande, J. J. 2003. La Valoración del Desarrollo Sostenible: Una propuesta metodológica (M. A. Andalucía Ecológica Ed.). Sevilla.

Saisana, M., Saltelli, A., \& Tarantola, S. 2005. Uncertainty and sensitivity analysis techniques as tools fot the quality assessment of composite indicators. J. R. Statist. Soc. A, 168 Part 2, 307-323.

Saisana, M., \& Tarantola, S. 2002. State-of-the-art report on current methodologies and practices for composite indicator development. Retrieved from European Commission-JCR: Italy:

Salinas, E., \& La O, J. A. 2006. Turismo y sustentabilidad: de la teoría a la práctica en Cuba. Cuadernos de Turismo, Universidad de Murcia, 17, 201-221.

Saltelli, A. 2006. Composite indicators between analysis and advocacy. Social Indicators Research, 81, 65-77. doi:10.1007/s11205-006-0024-9

Saltelli, A. 2007. Composite Indicators between analysis and advocacy. Social Indicators Research, $8(1), 65-77$.

Sanchs, J. 2015. The Age of Sustainable Development. Press. New York.

Santamarina, B. (2008). Antropología y medio ambiente. Revisión de una tradición y nuevas perspectivas de análisis en la problemática ecológica. AIBR. Revista de Antropología Iberoamericana, 3(2), 144-184. 
Santos, J. G., \& Gesinaldo, A. C. 2018. Atividades turísticas e indicadores de sustentabilidade: Um estudo em um destino turístico brasileiro. Pasos, 16(1), 37.

Sharma, A., \& Olsen, M. D. 2005. Tourism Satellite Accounts: Implementation in Tanzania. Annals of Tourism Research, 32(2), 367-385.

Sharpe, A. 2004. Literature Review of Frameworks for Macro-indicators, Centre for the Study of Living Standards. Ottawa, Canada.

Singh, R. K., Murty, H. R., Grupta, S. K., \& Dikshit, A. K. 2009. An overview of sustainability assessment methodologies. Ecological Indicators, 9, 189-212.

Slocombe, D. S. 1993. Environmental planning, ecosystem science, \& ecosystem approaches for integrating environment \& development. Environmental Management, 17(3), 289-303.

Torres, T., Sala Ríos, M., \& Farré Perdiguer, M. 2015. Grado de sostenibilidad de los ámbitos turísticos catalanes. PASOS. Revista de Turismo y Patrimonio Cultural. Special Issue, 13(6), 208.

U.N. 1998. Indicators of Sustainable Development Retrieved from http://www.un.org/esa/sustdev/indi6.htm

UNEP. 2011. Towards a Green Economy: Pathways to Sustainable Development and Poverty Eradication. Retrieved from Nairobi:

UNWTO. 2016a. International tourist arrivals up 4\% and reach a record 1.2 billion in 2015. UNWTO Word Tourism Barometer, 14, 1-6.

UNWTO. 2016b. Measuring Sustainable Tourism: Developing a statistical framework for sustainable tourism. UNWTO Statistics and Tourism Satellite Account Programme.

UNWTO. 2017. Comunicado de Prensa PR No. 17105. Retrieved from http://crm.unwto.org/sites/all/ modules/civicrm/extern/url.php?u=77846\&qid=20966502

Valentin, A., \& Spangenberg, J. H. 2000. A guide to community sustainability indicators. Environmental Impact Assessment Review, 381-392.

Van Broeck, E. 2005. Desarrollo Sustentable. Turismo, costas y educación., Universidad de Quintana Roo, México.

Volo, S. 2015. Indicators. The encyclopedia of sustainable tourism, 277-279.

Warhurst, A. 2002. Sustainability Indicators and Sustainability Performance Management. Report to the Project: Mining, Minerals and Sustainable Development (MMSD). Retrieved from Warwick, England: http://www.iied.org/sustainability_indicators.pdf

Wittemeyer, G., Elsen, P., Bean, W. T., Burton, A. C. O., \& Brashares, J. S. 2008. Acceleratedhumanpopulation growth at protected area edges. Science, 321, 123-126.

Yasarata, M., Altinay, L., Burns, P., \& Okumus, F. 2010. Politics and sustainable tourism development - Can they co-exist? Voices from North Cyprus. Tourism Management, 31(3), 345- 356.

Yuri Hanai, F. 2009. Sistema de indicadores de sustentabilidade: uma aplicação ao contexto de desenvolvimento do turismo na região de Bueno Brandão, Estado de Minas Gerais, Brasil. (Tese apresentada à Escola de Engenharia de São Carlos (EESC) da Universidade de São Paulo (USP) para obtenção do título de Doutor em Ciências da Engenharia Ambiental), Universidad de São Paulo, São Carlos, Estado de São Paulo.

Zarzosa, P. 1996. Aproximación a la medición del bienestar social. Retrieved from Valladolid:

Zarzosa, P., Molpeceres, M. M., Pérez, A., Prada, M. D., Prieto, M. M., C., R., \& Zarzosa, F. 2005. La calidad de vida en los municipios de la provincia de Valladolid. Diputación Provincial de Valladolid, Valladolid, España.

Zsögön, S. 2014. Antropología Ambiental: Conflictos por recursos naturculturales y vulnerabilidad de poblaciones. Madrid. 